\title{
Vortex polarity switching by a spin-polarized current
}

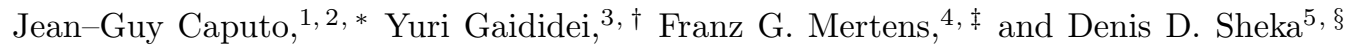 \\ ${ }^{1}$ Laboratoire de Mathématiques, INSA de Rouen, B.P. 8, 76131 Mont-Saint-Aignan cedex, France \\ ${ }^{2}$ Laboratoire de Physique theorique et modelisation, \\ Université de Cergy-Pontoise and C.N.R.S., Cergy-Pontoise, France \\ ${ }^{3}$ Institute for Theoretical Physics, 03143 Kiev, Ukraine \\ ${ }^{4}$ Physics Institute, University of Bayreuth, 95440 Bayreuth, Germany \\ ${ }^{5}$ National Taras Shevchenko University of Kiev, 03127 Kiev, Ukraine
}

(Dated: June 22, 2018)

\begin{abstract}
The spin-transfer effect is investigated for the vortex state of a magnetic nanodot. A spin current is shown to act similarly to an effective magnetic field perpendicular to the nanodot. Then a vortex with magnetization (polarity) parallel to the current polarization is energetically favorable. Following a simple energy analysis and using direct spin-lattice simulations, we predict the polarity switching of a vortex. For magnetic storage devices, an electric current is more effective to switch the polarity of a vortex in a nanodot than the magnetic field.

PACS numbers: 75.10.Hk, 75.40.Mg, 05.45.-a, 72.25.Ba, 85.75.-d
\end{abstract}

The control of magnetic nonlinear excitations (domain walls and vortices) via an electric current is of special interest for applications in spintronics [1, 2]. The spintransfer effect, theoretically predicted by Slonczewski [3] and Berger [4] and experimentally verified in [5, 6, 7], is very promising for magnetization switching with possible applications in magnetic storage devices. A perspective candidate for this program is the vortex (ground) state of a disk-shaped nanoparticle (nanodot), which provides high density storage and high speed magnetic RAM [8]

In this Letter we apply the spin-transfer effect to a nanodot in the vortex state. Using a continuum description for the magnetization dynamics we show that a spin current mainly acts like an effective magnetic field, applied to the sample along the $z$-axis. The influence of a perpendicular field on the vortex properties was studied in Refs. [9, 10] for easy-plane magnets. There it was shown that the vortex behavior depends on its core magnetization (polarity). A vortex with a magnetization pointing in the field direction (light vortex) has less energy than a vortex with a magnetization pointing away from the field direction (heavy vortex). For a strong enough field the heavy vortex loses its stability [10] and switches into a light vortex as confirmed by Monte Carlo simulations [11. The qualitative similarity between the current torque term and the magnetic field suggests that an appropriate current can switch the polarity of a vortex. Using a simple energy argument we show that the vortex polarity can be irreversibly switched by a spinpolarized current. These analytical predictions are confirmed by lattice spin dynamics. Since an electric current is easier to apply and less energetic than a magnetic field, we show that this is a more effective way of flipping the polarity of a vortex than the magnetic field.

To realize the program we use a pillar structure, first proposed in Ref. [12] (see Fig. 1). An electron current is injected in $\mathrm{FM}_{1}$ in $z$-direction, where it is polarized along $z$. The second ferromagnetic layer $\mathrm{FM}_{2}$ is separated from $\mathrm{FM}_{1}$ by a narrow (about $2-3 \mathrm{~nm}$ ) nonmagnetic layer NM 12, 13, 14, so the spin polarization of the current is conserved when it flows into $\mathrm{FM}_{2}$. A spin torque, acting on spins of the free layer $\mathrm{FM}_{2}$, causes their precession around $z$ with a constant out-of-plane component [3].

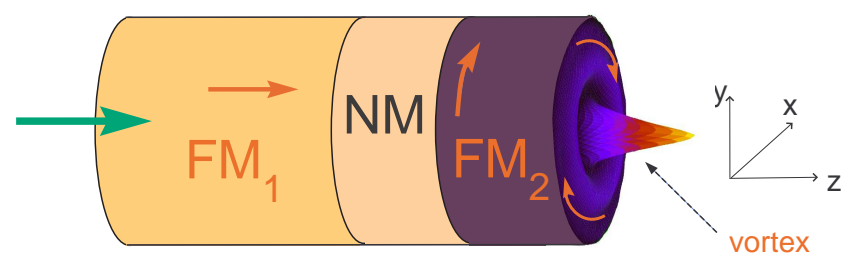

FIG. 1: (Color online). Schematic of the heterostructure.

The magnetization dynamics in the thin free layer $\mathrm{FM}_{2}$ is described by the Landau-Lifshitz-Gilbert equation with account of the spin-torque effect by a SlonczewskiBerger term [3, 那. This equation in dimensionless form is

$$
\begin{aligned}
\frac{\mathrm{d} \boldsymbol{S}_{\boldsymbol{n}}}{\mathrm{d} t}= & {\left[\boldsymbol{S}_{\boldsymbol{n}} \times \boldsymbol{F}_{\boldsymbol{n}}\right]-\varepsilon\left[\boldsymbol{S}_{\boldsymbol{n}} \times \frac{\mathrm{d} \boldsymbol{S}_{\boldsymbol{n}}}{\mathrm{d} t}\right] } \\
& +j a(\eta)\left[\boldsymbol{S}_{\boldsymbol{n}} \times\left[\boldsymbol{S}_{\boldsymbol{n}} \times \frac{\boldsymbol{\sigma}}{1+b(\eta) \boldsymbol{S}_{n} \cdot \boldsymbol{\sigma}}\right]\right],
\end{aligned}
$$

where $\boldsymbol{S}_{\boldsymbol{n}} \equiv\left(S_{\boldsymbol{n}}^{x}, S_{\boldsymbol{n}}^{y}, S_{\boldsymbol{n}}^{z}\right)$ is a classical spin vector with fixed length 1 on the site $\boldsymbol{n}$ of the lattice, $\varepsilon$ is a damping coefficient. The effective magnetic field is given by $\boldsymbol{F}_{n}=-\partial \mathcal{H} / \partial \boldsymbol{S}_{n}$, where $\mathcal{H}$ is the magnetic energy of the free layer. We consider the model of a classical easy-axis ferromagnet described by the following Hamiltonian

$$
\mathcal{H}=-\frac{1}{2} \sum_{\left(\boldsymbol{n}, \boldsymbol{n}^{\prime}\right)}\left(\boldsymbol{S}_{\boldsymbol{n}} \cdot \boldsymbol{S}_{\boldsymbol{n}^{\prime}}-\delta S_{\boldsymbol{n}}^{z} S_{\boldsymbol{n}^{\prime}}^{z}\right)+\mathcal{H}_{\mathrm{dd}}
$$


The summation runs over nearest-neighbor pairs $\left(\boldsymbol{n}, \boldsymbol{n}^{\prime}\right)$, $0<\delta<1$ is the anisotropy constant, $\mathcal{H}_{d d}$ is the magnetic dipole-dipole interaction. The unit vector $\boldsymbol{\sigma}$ gives the direction of the spin polarization ( along $z$ in our case), $j=J_{e} / J_{p}$ is the normalized spin current, $J_{e}$ is the electrical current density, $J_{p}=\mu_{0} M_{S}^{2}|e| d / \hbar$, where $d$ is the disk thickness, $e$ is the electron charge, $\mu_{0}$ is the vacuum permeability, $M_{S}$ is the saturation magnetization. The functions $a(\eta)$ and $b(\eta)$ have the form [3]

$a(\eta)=\frac{4 \eta^{3 / 2}}{3(1+\eta)^{3}-16 \eta^{3 / 2}}, \quad b(\eta)=\frac{(1+\eta)^{3}}{3(1+\eta)^{3}-16 \eta^{3 / 2}}$,

where $0<\eta<1$ is the degree of spin polarization.

In weakly anisotropic magnets with $\delta \ll 1$, the characteristic size of excitations $\ell_{\delta}=1 / \sqrt{z \delta}$ is larger than the lattice constant $a=1$ ( $z$ is the number of nearest neighbors in the lattice), so that one can use the continuum approximation for the Hamiltonian (2)

$$
\begin{aligned}
\mathscr{E} & =\mathscr{E}_{0}+\frac{1}{2} \int \mathrm{d} \boldsymbol{r}\left[(\boldsymbol{\nabla} \theta)^{2}+\sin ^{2} \theta(\boldsymbol{\nabla} \phi)^{2}\right. \\
& \left.+\frac{1}{\ell_{\delta}^{2}} \cos ^{2} \theta-\boldsymbol{H}_{\mathrm{dm}} \cdot \boldsymbol{S}\right]
\end{aligned}
$$

where $\mathscr{E}_{0}$ is a constant, $\boldsymbol{H}_{\mathrm{dm}}$ is an effective demagnetization field which stems from the dipoledipole interaction, and the parameterization $\boldsymbol{S}=$ $(\sin \theta \cos \phi, \sin \theta \sin \phi, \cos \theta)$ is used. It is well known 15, 16. that a flat cylindrical (disk-shaped) nanodot with a radius $R$ smaller than a critical value $R_{0}$ is in an inplane mono-domain state, whereas for $R>R_{0}$ the dot is in a vortex (curling) state. The vortex state for $R>R_{0}$ becomes energetically preferable as a result of the competition between the exchange and the dipole-dipole interactions. Typical values are $R_{0}=50 \mathrm{~nm}$ for a permalloy nanodisk with $d=15 \mathrm{~nm}[8]$. Generally, the demagnetization field is a nonlocal functional of $\boldsymbol{S}$, but for flat dots, i.e. for dots with the aspect ratio $d / R \ll 1$, the action of and the magnetostatic field can be presented in a localized form 17, 18]

$$
\boldsymbol{H}_{\mathrm{dm}}=-\frac{1}{\ell_{e}^{2}} \cos \theta \boldsymbol{e}_{z}
$$

where $\ell_{e}$ is the exchange length 19]; hence the demagnetization field is equivalent to an effective easyplane anisotropy with a typical magnetic length scale $\ell=\ell_{\delta} \ell_{e} / \sqrt{\ell_{\delta}^{2}+\ell_{e}^{2}}$.

Thus in the continuum limit the current driven dynamics of flat nanodots is described by the set of equations

$$
\begin{aligned}
\sin \theta \partial_{t} \phi & =-\frac{\delta \mathscr{E}}{\delta \theta}-\varepsilon \partial_{t} \theta, \\
-\sin \theta \partial_{t} \theta & =-\frac{\delta \mathscr{E}}{\delta \phi}-\varepsilon \sin ^{2} \theta \partial_{t} \phi+\frac{j a(\eta) \sin ^{2} \theta}{\sigma+b(\eta) \cos \theta},
\end{aligned}
$$

where the energy functional $\mathscr{E}$ is given by Eq. (3) with the demagnetization field given by Eq. (14) and $\sigma= \pm 1$ gives the sign of the spin polarization of the current.

Let us discuss the stationary solutions of Eqs. (5) without and with current first for homogeneous magnetization and then for a vortex state. In mono-domain nanodots for $j=0$ the magnetization is uniform and lies in the $x y$-plane. Equation (5) shows that a current causes a finite out-of-plane magnetization

$m_{0} \equiv \cos \theta_{0}=\frac{\sigma}{2 b}\left(\sqrt{1+\frac{4 j a b \ell^{2}}{\varepsilon}}-1\right), \quad \omega \equiv \partial_{t} \phi=\frac{m_{0}}{\ell^{2}}$,

where the in plane component $\phi$ rotates around the $z-$ axis with the frequency $\omega$. Because $\left|m_{0}\right|<1$ this state exists for small $j$, it is linearly stable for $\left|j a \ell^{2}-\varepsilon b\right|<\varepsilon$. Together with this state there is always the fixed point $\theta_{0}=0\left(\right.$ resp. $\left.\theta_{0}=\pi\right)$ which is stable for $j a \ell^{2}-\varepsilon b>\varepsilon$ (resp. $j a \ell^{2}-\varepsilon b>-\varepsilon$ ).

In the vortex state the magnetization structure is determined by the expressions

$$
\cos \theta=p f(r), \quad \phi= \pm \frac{\pi}{2}+\chi,
$$

where $r$ and $\chi$ are the polar coordinates in the $x y$-plane. The localized function $f(r)$ describes the core of the vortex. In the core the magnetization is nearly perpendicular to the disk-plane and the index $p= \pm 1$, which is termed polarity [20], determines which way the out-ofplane structure is oriented. In nanodots for which the vortex (6) is the ground state a current leads to a stationary state where the in-plane spin component rotates with the angular frequency $\omega$. The in-plane and out-ofplane fields can be written as

$$
\theta=\theta(r), \quad \phi= \pm \frac{\pi}{2}+\chi+\omega t+\psi(r),
$$

and from (5) they follow the equations

$$
\begin{aligned}
& \nabla_{r}^{2} \theta+\frac{\sin \theta}{\ell^{2}}\left(\cos \theta-m_{0}\right)-\sin \theta \cos \theta\left[\frac{1}{r^{2}}+\left(\partial_{r} \psi\right)^{2}\right]=0, \\
& \nabla_{r}^{2} \psi+2 \cot \theta \partial_{r} \theta \partial_{r} \psi+\frac{\varepsilon b m_{0}}{\ell^{2}} \frac{\cos \theta-m_{0}}{\sigma+b \cos \theta}=0,
\end{aligned}
$$

where $\nabla_{r}^{2}=r^{-1} \partial_{r}\left(r \partial_{r}\right)$.

Thus, under the action of the current, the azimuthal angle $\phi$ acquires an additional dependence $\psi$ on the radial coordinate $r$ roughly proportional to $\varepsilon b m_{0} / \ell^{2}$. However, for weak damping $(\varepsilon \ll 1)$ and for small spin polarization $(\eta \ll 1)$ this additional dependence does not change significantly the spatial dependence of the out-of-plane component $\cos \theta$. In this limit, in the rotating frame of reference where $\phi$ is replaced by $\widetilde{\phi}=\phi+\omega t$, the Eqs. (5) take on the usual Landau-Lifshitz-Gilbert form without spin-torque term. A spin current in this case is equivalent to an effective magnetic field applied along the hard 

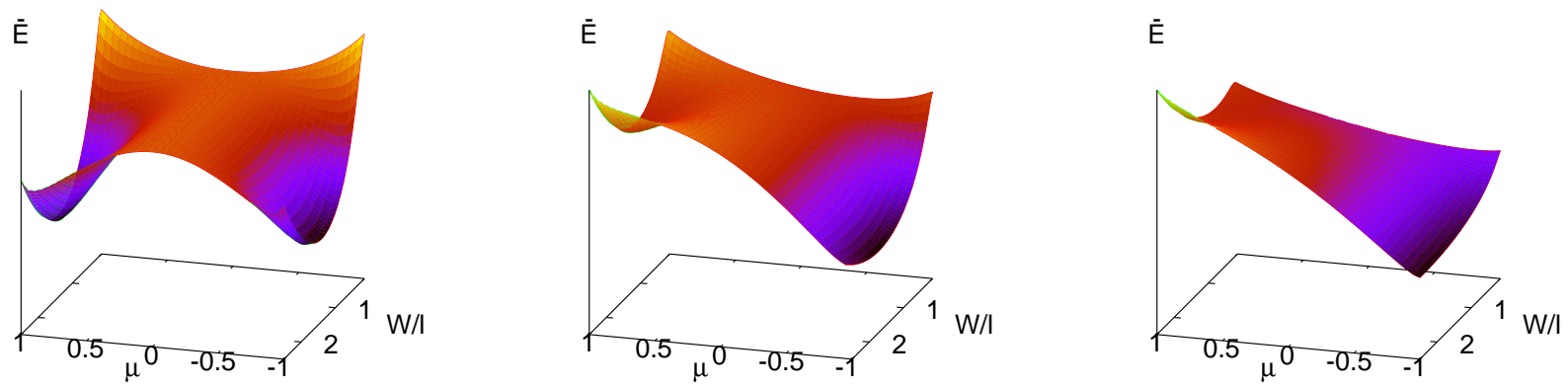

FIG. 2: (Color online). The energy of the vortex (9) for three different fields: $m_{0}=0$ (left panel), $m_{0}=-0.2$ (middle panel) and $m_{0}=-0.5$ (right panel). The cutoff parameter $a=0.264 a_{0}$ and $\ell=2.89 a_{0}$. The switching occurs for $m_{0} \approx-0.47$.

$z$-axis. The energy of the system in the rotating frame reads:

$$
\widetilde{\mathscr{E}}=\mathscr{E}-\int \omega \cos \theta \mathrm{d} \boldsymbol{r}
$$

and the model under consideration is similar to the socalled cone-state model 9, 10. It was shown in Refs. [9, 10] that the magnetic field removes the degeneracy with respect to the vortex polarity $p$. There are light (heavy) vortices, where the core is magnetized along (against) the field. The light vortex has always lower energy than the heavy one [9]. The range of anisotropy and magnetic field for which vortices are stable was found in Ref. 10. The above mentioned qualitative similarity between the current torque term and the magnetic field suggests that by applying an appropriate current one can achieve switching between the vortex states with different polarities. It is worth reminding that in the continuum limit the vortex states with different polarities are separated by an infinite barrier. However, in real systems with their discrete lattice structure, the barrier is finite 21] and switching can occur (see e.g. Refs. 22, 23, 24] where switching due to the noise or an ac magnetic field was investigated).

To give some insight into the current-induced switching mechanism, we propose here a simple continuum picture, where the discreteness effects are modeled by the cut-off parameter $a$, which is of the order of the lattice constant $a_{0}$. We introduce the simple two-parameters Ansatz

$$
\cos \theta=\left(\mu-m_{0}\right) e^{-r^{2} / W^{2}}+m_{0}, \quad \phi= \pm \frac{\pi}{2}+\chi,
$$

where the variational parameter $\mu$ characterizes the outof-plane core magnetization while the parameter $W$ gives the core width. Using this ansatz, one can rewrite the energy (7) in the form $\widetilde{\mathscr{E}} \approx \pi d\left[\left(1-m_{0}^{2}\right) \ln (L / a)+\mathcal{E}\right]$, where

$$
\begin{aligned}
\mathcal{E} & =2 \int_{0}^{\exp (-\xi)} \frac{t \ln t \mathrm{~d} t}{\left(t-\frac{1-m_{0}}{\nu}\right)\left(t+\frac{1+m_{0}}{\nu}\right)}-\nu m_{0} \mathrm{E}_{1}(\xi) \\
& -\frac{\nu^{2}}{2} \mathrm{E}_{1}(2 \xi)+\frac{\nu^{2} W^{2}}{4 \ell^{2}}, \quad \nu=\mu-m_{0}, \xi=\frac{a^{2}}{W^{2}} .
\end{aligned}
$$

Here $\mathrm{E}_{1}(\xi)$ is the exponential integral. Without the spincurrent, there exists a double-well potential, which provides equal minima for $\mu=p= \pm 1$ and $W=\ell \sqrt{2}$, see the left panel of Fig. 2. Such minima exist only if the cutoff parameter $a / \ell$ is smaller than some critical value which corresponds to the transition between out-of-plane and in-plane vortices 21. For the square lattice such a transition is realized for $\delta=0.297$ [21] or $\ell \approx 0.918 a_{0}$; this corresponds to $a / \ell \approx 0.288$ and $a \approx 0.264 a_{0}$.

If the current is turned on, one of the vortex states, namely the vortex polarized along $m_{0}$, becomes energetically preferable, see the middle panel of Fig. 2. When the current exceeds some critical value, the barrier disappears, and the switching occurs, see the right panel of Fig. 2. In terms of this variational Ansatz, the switching phenomenon can be considered as the motion of an effective mechanical particle in the two-dimensional potential (9). For small currents the particle is captured in one of the potential minima but when the current exceeds a threshold value one of the minima disappears and the particle moves to the another one. The vortex core width $W \approx \ell \sqrt{2\left(p+m_{0}\right) /\left(p-m_{0}\right)}$ increases with the current for light vortices and decreases for heavy ones, which agrees with results for the static field [9]. The critical value of $m_{0}$, where switching occurs, depends on the value of a cut-off parameter, $a / \ell$ : for the parameters presented on Fig. 2, the critical value is $m_{0} \approx-0.47$.

To validate our analytical predictions, we performed spin-lattice simulations. We stress that the standard micromagnetic simulations are not adequate for this 


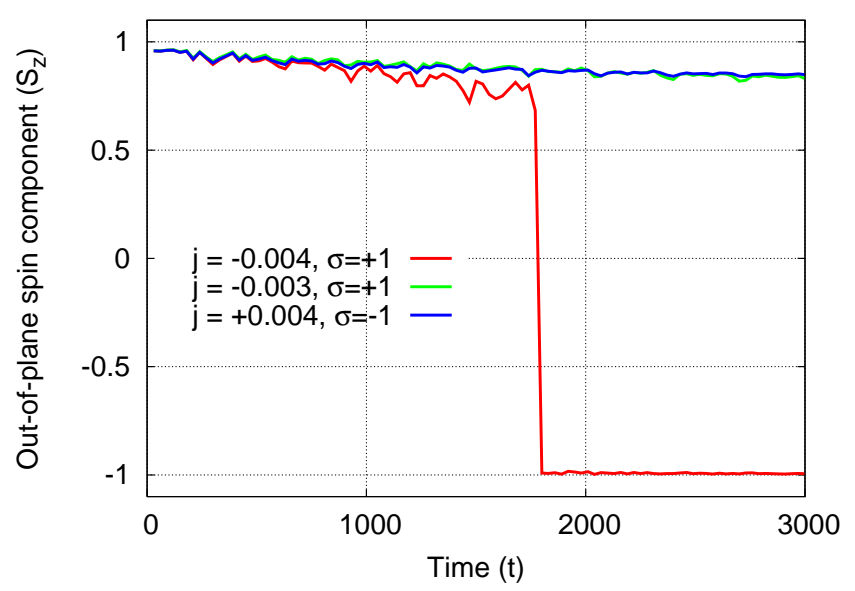

FIG. 3: (Color online). Amplitude of $S_{z}(t)$ at the vortex center from numerical simulations on a square lattice with circular boundary of diameter $L=200$. Other parameters of the system: $\eta=0.25, \varepsilon=0.01, \delta=0.03$; time is measured in units of $\hbar / J$. The critical current $j \approx-0.0033$.

problem, because the switching phenomenon has a discrete nature, while micromagnetic simulations are a discretization of originally continuum Landau-Lifshitz equations. We have integrated numerically the discrete (spin-lattice) Eqs. (11) over square lattices of size $L \times L$ using a 4th-order Runge-Kutta scheme with time step 0.01 and free boundary conditions. The spin system is defined by a circular border with the diameter $L$. We have fixed an exchange easy-plane anisotropy parameter $\delta=0.03$ instead of the dipolar term, corresponding to $\ell / a_{0}=2.89$, and we used the damping parameter $\varepsilon=0.01$. We have considered system sizes in the range $L / a_{0} \in(40,200)$.

In our simulations we observed switching between heavy and light vortices as a result of the influence of the current. Typical pictures are presented in Fig. 3 . The critical current $j \approx-0.0033$ is in a good agreement with our continuum model calculations: the critical value of $m_{0} \approx-0.47$ (see Fig. 2), which corresponds to $j \approx-0.00332$.

To summarize, in this work the spin-transfer effect was investigated for the vortex state nanodot. The switching of the vortex core magnetization was studied analytically and confirmed numerically by direct spin-lattice simulations. Let us estimate the critical current for a nanodot. Using typical parameters for the permalloy disks 25] $\left(\eta=0.26, M_{S}=640 \mathrm{kA} / \mathrm{m}, \varepsilon=0.01, d \approx 10 \mathrm{~nm}\right)$, one can estimate that $J_{\mathrm{cr}} \approx 10^{10} \mathrm{~A} / \mathrm{m}^{2}$. The total current is about $10 \mathrm{~mA}$. For magnetic storage devices an electric current is then more effective to switch the polarity of a vortex in a nanodot than the magnetic field which is about the $0.1 \div 1 \mathrm{~T}$.

The authors thank S. Demokritov (Münster University) for useful discussions. Yu.G. and D.D.Sh. thank the University of Bayreuth, where part of this work was per- formed, for kind hospitality and acknowledge the support from Deutsches Zentrum für Luft- und Raumfart e.V., Internationales Büro des BMBF in the frame of a bilateral scientific cooperation between Ukraine and Germany, project No. UKR 05/055. J.G.C., Yu.G. and D.D.Sh. acknowledge support from a Ukrainian-French Dnipro grant (No. 82/240293). D.D.Sh. acknowledges the support from the Alexander von Humboldt-Foundation.

* Electronic address: caputo@insa-rouen.fr

$\dagger$ Electronic address: yugaid@uni-bayreuth.de

¥ Electronic address: franz.mertens@uni-bayreuth.de

$\S$ Electronic address: denis_sheka@univ.kiev.ua

[1] Y. Tserkovnyak, A. Brataas, G. E. W. Bauer, and B. I. Halperin, Reviews of Modern Physics 77, 1375 (pages 47) (2005),

[2] S. D. Bader, Reviews of Modern Physics 78, 1 (pages 15) (2006), .

[3] J. C. Slonczewski, J. Magn. Magn. Mater. 159, L1 (1996),

[4] L. Berger, Phys. Rev. B 54, 9353 (1996),

[5] M. Tsoi, A. G. M. Jansen, J. Bass, W.-C. Chiang, M. Seck, V. Tsoi, and P. Wyder, Phys. Rev. Lett. 80, 4281 (1998).

[6] E. B. Myers, D. C. Ralph, J. A. Katine, R. N. Louie, and R. A. Buhrman, Science 285, 867 (1999).

[7] I. N. Krivorotov, N. C. Emley, J. C. Sankey, S. I. Kiselev, D. C. Ralph, and R. A. Buhrman, Science 307, 228 (2005).

[8] R. P. Cowburn, J. Magn. Magn. Mater. 242-245, 505 (2002), .

[9] B. A. Ivanov and D. D. Sheka, Low Temp. Phys. 21, 881 (1995), .

[10] B. A. Ivanov and G. M. Wysin, Phys. Rev. B 65, 134434 (pages 17) (2002),

[11] K. W. Lee and C. E. Lee, Phys. Rev. B 70, 144420 (pages 4) (2004), .

[12] A. D. Kent, B. Ozyilmaz, and E. del Barco, Appl. Phys. Lett. 84, 3897 (2004), .

[13] H. Xi, K.-Z. Gao, and Y. Shi, J. Appl. Phys. 97, 044306 (pages 4) (2005), .

[14] H. Xi, Y. Shi, and K.-Z. Gao, Phys. Rev. B 71, 144418 (pages 5) (2005),

[15] N. A. Usov and S. E. Peschany, J. Magn. Magn. Mater. 118, L290 (1993),

[16] R. P. Cowburn and M. E. Welland, Science 287, 1466 (2000).

[17] G. Gioia and R. D. James, Proc. R. Soc. Lond. A 453, 213 (1997), .

[18] B. A. Ivanov and C. E. Zaspel, Appl. Phys. Lett. 81, 1261 (2002).

[19] R. Skomski, J. Phys. C 15, R841 (2003),

[20] D. L. Huber, Phys. Rev. B 26, 3758 (1982), .

[21] G. M. Wysin, Phys. Rev. B 49, 8780 (1994), .

[22] Y. Gaididei, T. Kamppeter, F. G. Mertens, and A. Bishop, Phys. Rev. B 59, 7010 (1999), .

[23] Y. Gaididei, T. Kamppeter, F. G. Mertens, and A. R. Bishop, Phys. Rev. B 61, 9449 (2000).

[24] J. P. Zagorodny, Y. Gaididei, F. G. Mertens, and A. R. 
Bishop, Eur. Phys. J. B 31, 471 (2003).

[25] M. A. Hoefer, M. J. Ablowitz, B. Ilan, M. R. Pufall, and
T. J. Silva, Phys. Rev. Lett. 95, 267206 (pages 4) (2005), 